\title{
XV: Reactive Spaces
}

\author{
Esteban Garcia Bravo \\ Purdue University \\ Knoy Hall, 401 N. Grant Street \\ West Lafayette, IN 47907, USA \\ garcia0@purdue.edu
}

\author{
Jorge A. Garcia \\ Purdue University \\ Knoy Hall, 401 N. Grant Street \\ West Lafayette, IN 47907, USA \\ garci191@purdue.edu
}

\author{
Aaron Zernack \\ Castle Bravo Tapes \\ 215 N. 4th Street \\ Lafayette, IN 47901, USA \\ aaronzernack@gmail.com
}

\section{INTRODUCTION}

Fifteen points $(\mathrm{XV})$ is an interactive floor projection in which viewers are encouraged to move throughout the space and engage in the manipulation of the art piece. The spaces generated through these fifteen points detect the body of the participant, bending and distorting the architecture of the piece. When a presence is no longer sensed, the shapes are autonomously restored with a burst of energy to their original state, while a generative soundscape orchestrates the entire experience.

This piece is the result of a collaborative effort in sound composition, computational art and mathematics. Integrating computer vision technologies and a nouvelle interface of Control Voltage audio synthesis (CV/Gate) with Bluetooth communication, $\mathrm{XV}$ expands on prior body of work in interactive visualization and sonification. The installation immerses the viewer in a synaesthetic environment; visual forms are produced as an analogue to music (Betancourt 2013).

\section{EMBODIED MODERNISM}

A point of inspiration for our development was the Neo-Concrete movement, a short-lived group from Brazil in late 1950s. These artists departed from a systematic approach to geometric abstraction from the earlier "Concrete" movement, who focused on constructive aesthetic. The Neo-concretes did not completely reject geometry in their compositions, but rather incorporated it in a more playful way. One of the key elements of Neo-Concrete art was the belief that a piece can only be completed with the embodied participation of a viewer.

One example of such participation was Bichos by Lygia Clark. The Bichos are objects that can be manipulated by the viewer, moving corners and vertices to redefine the shape of the piece. In Parangolés, a series of Neo-Concrete happenings by Helio Oiticica, there was live music involved to make more emphasis on an experiential and multisensory approach to art. In Parangolés, the participants manipulated colored geometries made out of fabric.

These ideas of the experiential with geometric spaces triggered our interest. New technologies have allowed us to create reactive and participative systems that integrate Neo-Concrete utopias further.

\section{SENSING THE BODY}

Our installation was created through an array of video mapped surfaces. Each surface consists of an irregular polygon defined by sets of points. The motion capture algorithm takes into consideration the position of the user in relation to the design.

For sensing motion, we used an infrared camera installed next to the projector in the ceiling. They are both facing down towards the floor. The casted shadows in the floor are analyzed using the OpenCV library, tracking the position of the participants as blobs. The location of the participant is stored and compared in relation to their position in the geometric space. Since the areas of the video projection and the space the infrared camera is sensing may differ, we use simple linear interpolation to accurately map the position of the user in the projected space.

For this project, we detected the location of the user as a vector in space. In the algorithm, a "horizontal ray" is traced in relation to each of the polygonal areas. This method is known as the IPT method and is described in a recent paper by $\mathrm{Li}$, $\mathrm{He}$ and Tian (2012). According to the IPT method, if the vector intersects the edge of the polygon an odd number of times, it means that the vector it is inside the polygon. Conversely, if the vector intersects the edge of a polygon an even number of times, then it means that it is outside the irregular polygon (Figure 1). 


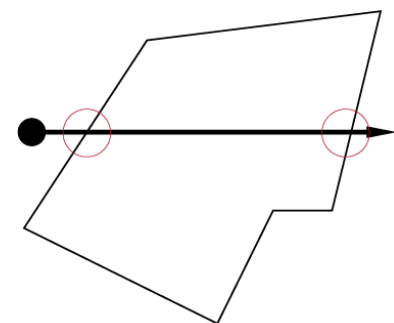

Even number of cuts $=$ User is outside

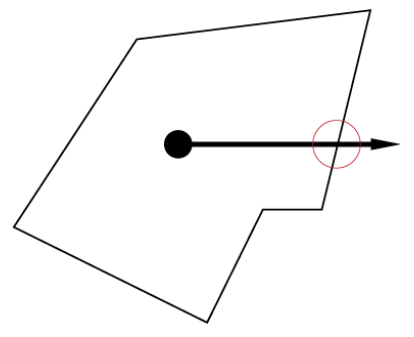

Odd number of cuts $=$ User is inside
Figure 1: Illustration of the IPT method

\section{FUSION OF THE SENSES}

The accompanying sound composition contributes greatly to the generative element of the system because it induces a form of auditory-visual synaesthesia in participants. The installation features a newly developed protocol called Geometry Synth, developed by a designer known as Minigorille (2014). This device communicates CV (modular sound synthesis) data to a remote computer via bluetooth communication. The sampled data is then visualized through a Processing application, and the sound variables are reinterpreted in the form of visual changes such as the amount of color or intensity of the experience. In this way, the soundscape is an integral part of the entire experience as it enables a relation to one sense by the stimulation of another sense.

\section{CONCLUSION}

The interdisciplinary efforts in sound composition, mathematics and art allowed for a sensorially-rich and synaesthetic experience. XV allows viewers to be immersed in a shifting environment affected by their own bodies. This project explored geometric abstraction thorough an interactive and audiovisual platform.

\section{REFERENCES}

Betancourt, M. (2013) The History of Motion Graphics. Wildside Press, p. 12.

Li, Z., He, Y., \& Tian, Z. (2012) Overlapping Area Computation between Irregular Polygons for its Evolutionary Layout based on Convex Decomposition. Journal of Software, 7(2):485-492. doi:10.4304/jsw.7.2.485-492.

Minigorille (2014) Geometry Synthesizer. http://www.minigorille.com/geometry synth.html (retrieved 20 March 2015).

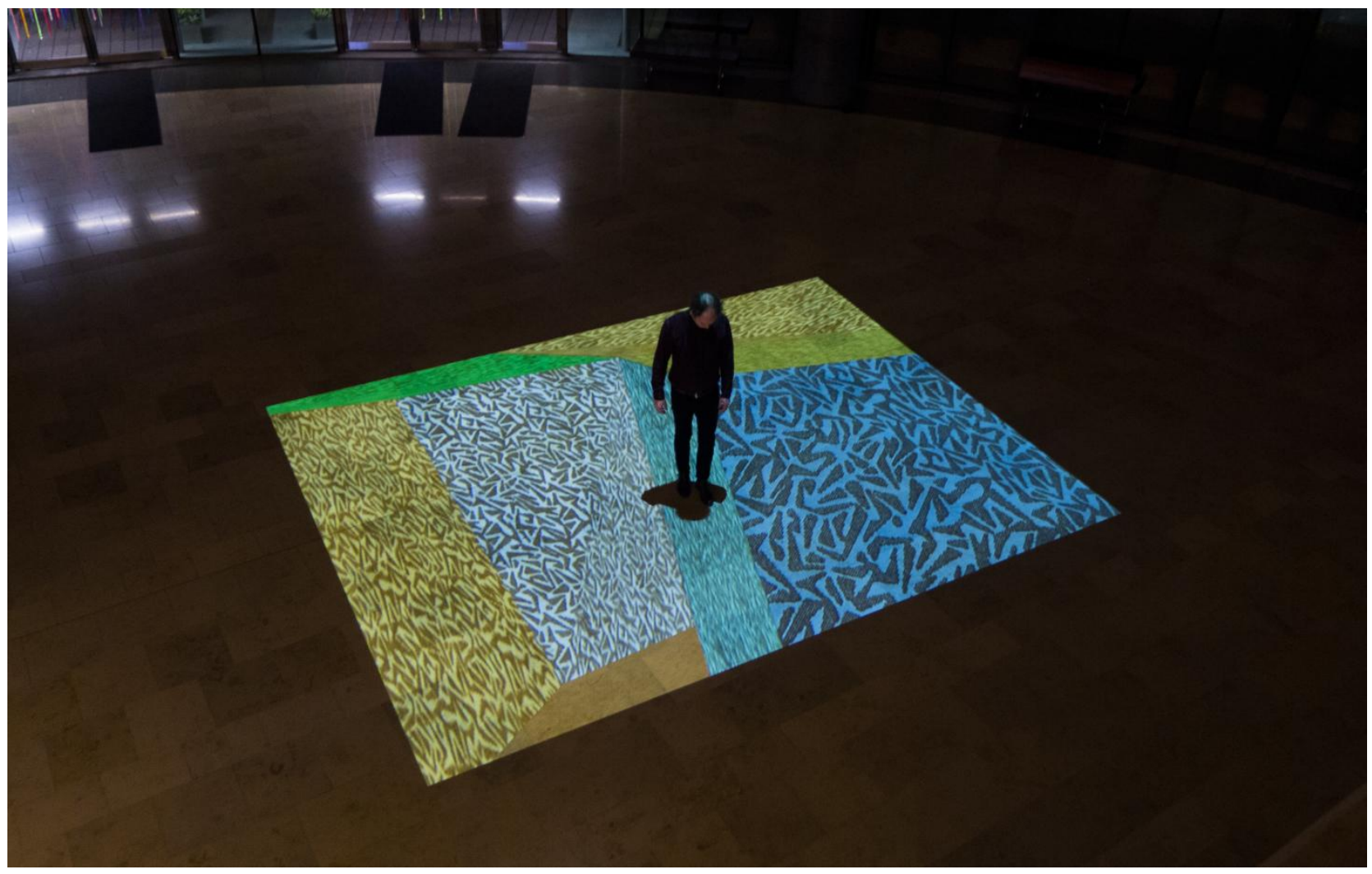

Figure 2: Image of the XV Installation 